\title{
The Relationships between the Pain Beliefs and Coping Strategies of Palliative Care Patients
}

\author{
Tuğba Menekli $\mathbb{D}^{1}$, Runida Doğan $\mathbb{D}^{2}$, Çiğdem Erce $\mathbb{D}^{3}$, Derya Atik $\mathbb{D}^{4}$ \\ 'Department of Nursing, Malatya Turgut Özal University Faculty of Health Sciences, Malatya, Turkey \\ ${ }^{2}$ Department of Surgical Nursing, Inönü University Faculty of Nursing, Malatya, Turkey \\ ${ }^{3}$ Malatya Training and Research Hospital, Palliative Care Unit, Malatya, Turkey \\ ${ }^{4}$ Division of Nursing, Osmaniye Korkut Ata University Faculty of Helath Sciences, Osmaniye, Turkey
}

ORCID iDs of the authors: T.M. 0000-0002-829I-8274; R.D. 0000-0003-3II3-8099; Ç.E. 0000-0002-4982-6204; D.A. 0000-0002-84970105.

Cite this article as: Menekli T, Doğan R, Erce Ç, Atik D. The Relationships between the Pain Beliefs and Coping Strategies of Palliative Care Patients. Cyprus J Med Sci. 2021; 6(3): 201-207.

\section{BACKGROUND/AIMS}

This descriptive study aims to determine the relationships between the pain beliefs and coping strategies of palliative care patients.

\section{MATERIAL and METHODS}

This study was conducted in the Malatya Training and Research Hospital Palliative Care Clinic from March 10, 2019 to June 10, 2019. The sample consisted of 138 inpatients. The data were collected using a personal information form, the Pain Beliefs Questionnaire, the Pain Coping Questionnaire, and the Palliative Performance Scale. Statistical Package for the Social Sciences (SPSS) version I8.0 (IBM SPSS Corp.; Armonk, NY, USA) software was used to analyze the data. The required ethical approvals were obtained.

\section{RESULTS}

The patients' mean age was $59.01 \pm 9.38$. Of them, $65.1 \%$ were female, and $89.7 \%$ were married. Of the patients, $56.3 \%$ had completed primary school, and $75.3 \%$ were unemployed. Of the patients, $70.5 \%$ believed that pain is controlled by God. Their mean organic beliefs score was $3.04 \pm 0.38$, and their mean psychological beliefs score was $4.83 \pm 1.00$. Their mean scores for self-management, helplessness, conscious cognitive attempts, and medical remedies were $19.07 \pm 5.18,10.72 \pm 3.00,13.85 \pm 4.05$, and $11.54 \pm 3.08$, respectively. Their organic beliefs scores had a negative relationship with self-management scores $(P<.001, r=-392)$ and conscious cognitive attempts scores $(P<.001, r=-.350)$, and they had a positive weak relationship with helplessness scores $(P<.001, r=.380)$.

\section{CONCLUSION}

The pain beliefs of patients in palliative care affect their ability to cope with pain. The planning and implementation of nursing pain management interventions should consider the relationships between the pain beliefs and pain coping strategies of patients.

Keywords: Palliative care, pain beliefs, coping with pain, nursing

\section{INTRODUCTION}

Palliative care is a multidisciplinary approach that starts with diagnosis and continues during and after treatment. Palliative care helps patients to cope with a variety of disease-related symptoms.' When the symptoms are brought under control, patients' adaptation to their community increases, their recovery period begins, and they become able to tolerate their treatments' side effects like vomiting and dizziness, etc. ${ }^{2,3}$ Palliative care aims to provide patients with an active and quality life until their death., 2 Pain is a critical symptom that affects the course of diseases, and treating it is a priority issue. 2,4

Inadequate pain management leads to serious complications and increases morbidity and mortality and the length of hospital stays. ${ }^{5}$ It reduces patients' quality of life by affecting their conduct of daily activities. ${ }^{4}$ Pain and its effects are significant causes of stress. Cognitive and behavioral responses to stress that are considered "coping with pain" affect the severity of pain, pain-related disabilities, and patient psychology. ${ }^{6,7}$ Coping is defined as people's resistance to events or factors that cause stress and their cognitive, emotional, and behavioral responses to endure them. Coping 
strategies vary with a variety of factors such as age, gender, culture, and disease. They are unique to each individual. ${ }^{6,8,9}$ Pain includes sensory, emotional, and behavioral factors. It is a multidimensional experience that has been explained using a variety of models, including the pathogenic and biopsychosocial models. The pathogenic model of pain focuses on whether pain is the result of a direct tissue trauma or a physiological destruction. The biopsychosocial model involves cognitive, emotional, and behavioral factors. These factors are reported to affect pain response indirectly by affecting patients' neurochemistry. ${ }^{\mid 0, I I}$

Belief is a cognitive factor that is based on social psychology. It is seen as one of the main constituents of people's thought systems in the cognitive perspective. Pain beliefs involve organic and psychological beliefs. Organic beliefs are based on the perception that pain is caused by bodily injury or harm. People believe that an increase in the level of injury increases pain. This makes activity/exercise and a biomedical approach important for eliminating the problem causing the pain. Psychological beliefs are based on the perception that pain is affected by psychological factors (anxiety, depression, etc.). A variety of techniques such as relaxation and distraction can be used for pain management. ${ }^{8,10,12}$ Studies of pain beliefs show that patients' treatments and coping strategies vary by their beliefs. ${ }^{8,1}$

It is important for nurses as members of multidisciplinary teams to detect the pain management problems of patients that related to their pain beliefs and to plan nursing pain management interventions that consider them. ${ }^{8,9}$ No studies of the relationships between the pain beliefs and pain coping strategies of patients in palliative care were found in the literature. Determining the pain beliefs of patients may help in the selection of the best coping method, and thus an effective pain management method. . II $^{-}$Therefore, our study aimed to evaluate the correlation between pain beliefs and coping strategies in palliative care patients. Other purposes of the research are to examine whether sociodemographic variables affect pain belief and coping with pain.

\section{MATERIALS and METHODS The Study's Population and Sample}

This descriptive study was conducted in the Malatya Training and Research Hospital Palliative Care Clinic from March 10,

\section{Main Points}

- Studies of pain beliefs show that patients' treatments and coping strategies vary by their beliefs.

- The patients' self-management scores had a positive strong relationship with conscious cognitive attempts scores and a negative weak significant relationship with helplessness scores.

- The female patients had higher self-management, conscious cognitive attempts, helplessness, and medical remedies scores. The female patients had higher psychological beliefs scores, and the male patients had higher mean organic beliefs scores.

- The cancer patients had lower self-management scores than the cardiovascular disease patients.
2019 to June 10, 2019, the only hospital in the city of Malatya that admits patients to palliative care. A necessary sample size of I40 people was determined using G*Power software and an effect size of 2.25 , a significance level $(\alpha)$ of 0.05 , and $80 \%$ power. The sample consisted of 138 patients.

\section{Inclusion Criteria}

Patients who were 18 years old and above, conscious, had no communication difficulties or mental disorders, scored $40 \%$ or higher on the Palliative Performance Scale (PPS), and agreed to participate were included in the study. The minimum palliative performance score for patients was $40 \%$. The criteria also included being conscious and having a normal or less than normal food intake, requiring major assistance for self-care, being unable to do most activities, having extensive disease, and being mainly in bed. Patients who scored below $40 \%$ on the PPS were excluded from the study because they would be tired due to their lack of functional capacity, and reliable data would not be obtained from them due to their unsteady levels of consciousness. ${ }^{13}$

\section{Data Collection}

The patients were informed about the study and told that its data would not be shared with third parties. The researchers collected the data in face-to-face interviews. The questionnaire and scale items were read, and the patients' responses were recorded by the researchers. Filling out the forms took roughly 30 minutes for each patient. The data were collected using a personal information form, the Pain Beliefs Questionnaire $(P B Q)$, the Pain Coping Questionnaire (PCQ), and the PPS.

The personal information form. It was created by researchers after a review of the literature. It consists of 13 questions about the patients' sociodemographic characteristics (age, gender, marital status, educational level, working status, caregiver, and diagnosis) and pain-related information (site of pain, severity of pain, pain history, type of analgesic, and the patient's beliefs about who controls their pain).

The Pain Beliefs Questionnaire. The PBQ was developed by Edward et al. ${ }^{14}$ to evaluate beliefs about the causes and treatment of pain. Berk ${ }^{15}$ did the validity and reliability study of the questionnaire's Turkish version. The PBQ has 12 items that concern organic and psychological pain beliefs. The organic beliefs subtest concerns the organic causes of pain. The psychological beliefs subtest is about psychological factors that affect the experience of pain. There is no cutoff point for the scores. Higher subscale scores indicate more pain beliefs, and lower subscale scores indicate less pain beliefs. The highest possible scores on the organic and psychological beliefs subscales are 6 , and the lowest possible scores are I. Cronbach's $\alpha$ of the Turkish versions internal consistency coefficients was 0.71 for the organic beliefs subtest and 0.73 for the psychological beliefs subtest in its reliability study. ${ }^{15}$

The Pain Coping Questionnaire. The PCQ was developed by Kleinke ${ }^{16}$ to determine pain-related patterns of affection and behavior. Hocaoğlu et al. ${ }^{6}$ did the validity and reliability study of its Turkish version. The PCQ has these subscales: selfmanagement, helplessness and conscious cognitive attempts, and medical remedies. It is a 4-point Likert-type scale ( $0=$ never to 3 = often) with 29 items. There is no cutoff point for the scores. The highest possible scores on the subscales of self- 
Table I. The Patients' Mean PBQ and PCQ Scores

\begin{tabular}{|c|c|c|c|c|}
\hline Scales & & Minimum & Maximum & Mean \pm SD \\
\hline \multirow{2}{*}{ Pain Coping Questionnaire } & Helplessness & 2.00 & 22.00 & $10.72 \pm 3.00$ \\
\hline & Conscious Cognitive Attempts & 3.00 & 20.00 & $13.85 \pm 4.05$ \\
\hline \multirow[t]{2}{*}{ Pain Beliefs Questionnaire } & Organic Beliefs & 1.96 & 5.59 & $3.04 \pm 0.38$ \\
\hline & Psychological Beliefs & 1.00 & 5.70 & $4.83 \pm 1.00$ \\
\hline
\end{tabular}

management, helplessness and conscious cognitive attempts, and medical remedies are 36,24 , and 27 , respectively. The lowest possible score on all the subscales is 0 . The Cronbach's $\alpha$ of the Turkish versions internal consistency coefficient was 0.75 in the questionnaire's reliability study. ${ }^{6}$

\section{The Palliative Performance Scale}

The PPS was developed by Anderson et al. ${ }^{13}$ to evaluate patients' ambulation, activity and evidence of disease, selfcare, food intake, and consciousness levels. PPS levels range from 0 to $100 \%$ in 10\% increments. They are determined by starting with ambulation in the leftmost column. After the most appropriate ambulation level is determined, other four columns are evaluated. Then, the overall best fit for patients is assigned as their PPS score. It should be noted that the left-hand columns are more determinant than the right-hand columns.

\section{Ethical Considerations}

Prior to the study, ethics committee approval and written permission were obtained from the Inönü University Noninvasive Clinical Research Ethics Committee (approval number: 2019/36-04) and the Malatya Training and Research Hospital. Written and verbal consent were obtained from the participants.

\section{Data Analysis}

The data were analyzed using Statistical Package for the Social Sciences (SPSS) version 18.0 (IBM SPSS Corp; Armonk, NY, USA) software and descriptive statistics (numbers, percentages, means, and standard deviations). The data were compared using the Mann-Whitney $U$ test and the Kruskal-Wallis test. Spearman's correlation was used to determine the relationships between variables. The confidence interval was $95 \%$, and the threshold for significance was $<5 \%$.

\section{RESULTS}

The patients' mean age was $59.01 \pm 9.38$. Of the patients, $65.1 \%$ were female, and $89.7 \%$ were married. Of them, $56.3 \%$ had completed primary school, and $75.3 \%$ were unemployed. Of the patients, $82.1 \%$ said that their caregivers were their family members. Of the patients, $63.5 \%$ were diagnosed with cancer, $24.5 \%$ were diagnosed with cardiovascular diseases, and $12.0 \%$ were diagnosed with neurological diseases. For the treatment of pain, $52.6 \%$ of patients used nonsteroidal anti-inflammatory drugs (NSAIDs), $20.3 \%$ used weak or strong opioids, and $27 \%$ used adjuvant drugs. Of the patients, $75.2 \%$ said that they had previously had pain complaints for other reasons. Of the patients, 57.3\% had back-lower back pain, 26.9\% had headneck pain, and $15.8 \%$ had arm-shoulder pain. Of the patients, $40.4 \%$ had severe levels of pain, $30.2 \%$ had very severe levels of pain, and $29.4 \%$ had moderate levels of pain. Of the patients, $70.5 \%$ believed that pain is controlled by God and $14.6 \%$ believed that pain is controlled by doctors. Another $10.3 \%$ believed that they controlled their own pain, and only $4.6 \%$ believed that pain is controlled by nurses.

Table I shows the patients' mean total and subscale PBQ and PCQ scores.

The patients' self-management scores had a positive strong relationship with conscious cognitive attempts scores $(P<.001$, $r=.675)$ and a negative relationship with helplessness scores $(P<.001, r=-.553)$. The patients' helplessness scores had a negative relationship with their conscious cognitive attempts scores $(P<.001, r=-.199)$ and a positive relationship with their medical remedies scores $(P<.001, r=.572)$. A positive relationship was found between their conscious cognitive attempts scores and medical remedies scores $(P<.001, r=.302)$. The patients' organic beliefs scores had a negative relationship with their self-management scores $(P<.001, r=-.392)$ and conscious cognitive attempts scores $(P<.00 \mathrm{l}, \mathrm{r}=-.350)$ and had a positive weak relationship with their helplessness scores $(P<$ $.001, r=.380$ ). A positive weak relationship was found between the patients' psychological beliefs scores and selfmanagement scores $(P<.05, r=.197)$. No significant relationship was found between the patients' organic beliefs and psychological beliefs $(P>.05, r=.062)$ (Table 2$)$.

The female patients had higher self-management, conscious cognitive attempts, helplessness, and medical remedies scores $(P<.0 \mathrm{l})$. The female patients had higher psychological beliefs scores, and the male patients had higher mean organic beliefs scores $(P<.05)$. The patients' age, marital status, education levels, and income levels had no significant relationship with their organic and psychological pain beliefs scores and PCQ scores $(P>$.05). The patients whose pain management was supported by people they lived with had higher scores for psychological pain beliefs, self-management, conscious cognitive attempts, helplessness, and medical remedies $(P<.05)$.

The cancer patients $(P<.01)$ had lower self-management scores than the cardiovascular disease patients. The cancer patients $(P<.001)$ had lower conscious cognitive attempts scores than the patients diagnosed with neurological diseases and higher medical remedies scores than the other groups $(P<$ .05). No significant difference was found between patients' diagnoses and their organic and psychological beliefs $(P>.05)$.

A significant difference was found between self-management $(P<.0 \mathrm{I})$, helplessness $(P<.0 \mathrm{I})$, and organic beliefs $(P<.05)$ scores of the patients who used opioids to treat pain. A significant difference was found between the patients' helplessness $(P<.00 \mathrm{l})$ and medical remedies $(P<.0 \mathrm{l})$ scores and their pain history. No significant difference was found between patients' 
Table 2. Correlations Between the Patients' PBQ and PCQ Scores

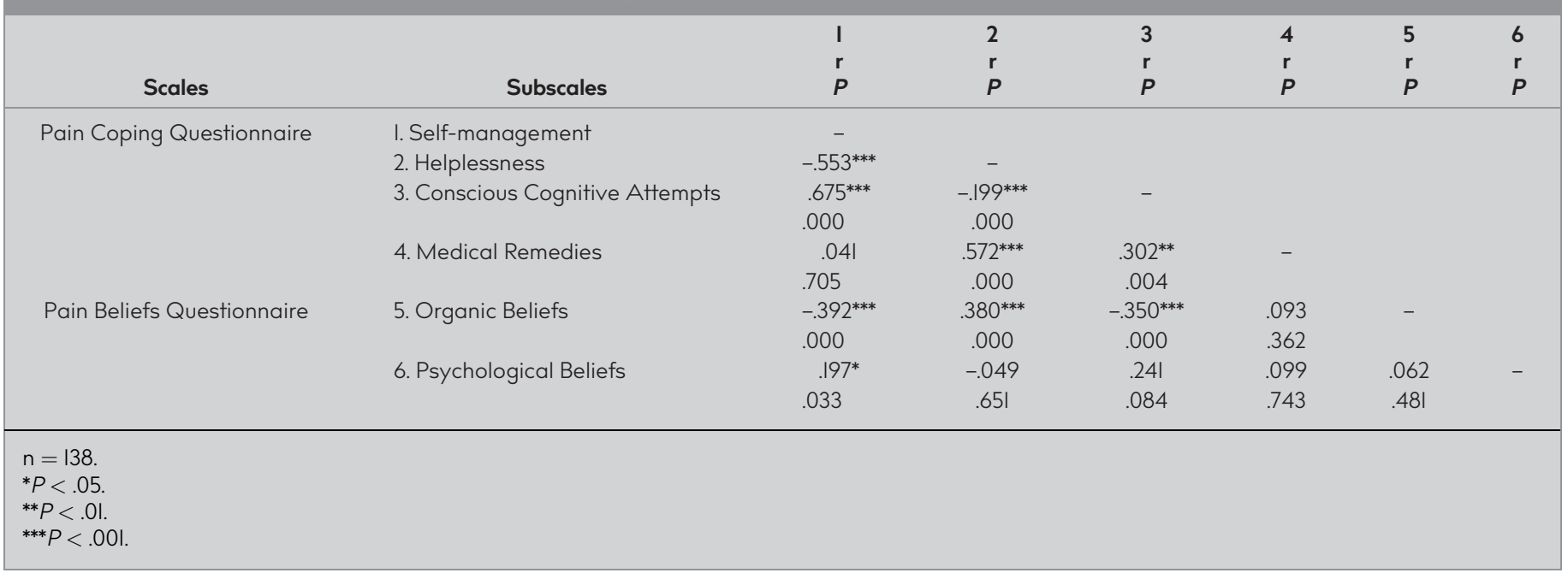

organic and psychological beliefs scores and their pain history $(P>.05)$ (Table 3).

There was a significant difference between the pain location and the scores of for self-management and conscious cognitive attempts $(P<.00 I)$. The patients who had head-neck pain had lower mean scores for self-management and conscious cognitive attempts. A significant difference was found between the severity of pain and the subscales of self-management $(P<$ $.00 \mathrm{l})$, helplessness $(P<.0 \mathrm{I})$, and medical remedies $(P<.0 \mathrm{I})$. The patients who had moderate levels of pain had higher mean scores for self-management. The patients who had very severe levels of pain had higher mean scores for medical remedies, and the patients who had intolerable levels of pain had higher mean scores for helplessness. A difference was found between the belief that patients control their own pain and organic beliefs scores $(P<.05)$. A significant difference was only found in the medical remedies scores of patients who believed that pain is controlled by nurses $(P<.05)$. There is a significant difference only in the helplessness scores of the patients who believe that pain control is in God $(P<.05)$ (Table 3).

\section{DISCUSSION}

Only one study that examines pain belief and coping with pain together was found in the literature. For this reason, a limited number of resources are used in this discussion of the results.

The patients' organic beliefs mean score was $3.04 \pm 0.38$, and their mean psychological beliefs score was $4.83 \pm 1.00$. Studies of pain beliefs have reported that organic and psychological beliefs scores vary with different samples (old people, patients in algology clinics, students, etc.). $8,10,17,18$

In our study, scores for self-management, helplessness, conscious cognitive attempts, and medical remedies were $19.07 \pm$ $5.18,10.72 \pm 3.00,13.85 \pm 4.05$, and II.54 \pm 3.08 , respectively. Few studies of coping with pain were found in the literature. These studies report different levels of mean subscale scores. 8,18 When the relationship of the PCQ subscales with each other is examined, the similarity in the results is quite remarkable., 8 The differences in the results were caused by the effect of variables such as age, gender, diagnosis, education level, site of pain, and severity of pain-on-pain beliefs and coping with pain.

A negative weak significant relationship was found between self-management scores and helplessness scores. Two studies conducted with different sample groups have similar results. ${ }^{8,18}$ A negative relationship was found between helplessness scores and conscious cognitive attempts scores. Another study has similar results. ${ }^{8}$ A positive strong relationship was found between self-management scores and conscious cognitive attempts scores. Madenci et al. ${ }^{19}$ also found a significant relationship between self-management scores and conscious cognitive attempts scores. A positive weak significant relationship was found between the patients' helplessness scores and medical remedies scores. A positive weak significant relationship was found between the patients' conscious cognitive attempts scores and medical remedies scores. There are similar results in the literature. 8,18 Patients who feel helplessness in pain management may have difficulty in coping with pain by themselves and may fail in pain management, particularly in cognitive and behavioral strategies. ${ }^{8,18}$ The results of the study are similar to those in the literature.

The patients' organic beliefs scores had a negative relationship with their self-management scores and conscious cognitive attempts scores and a positive weak relationship with their helplessness scores. Similarly, Babadağ et al. ${ }^{8}$ found that higher organic beliefs scores lead to lower self-management and conscious cognitive attempts scores and higher helplessness scores. A positive weak relationship was also found between the patients' psychological beliefs scores and selfmanagement scores. ${ }^{8}$ A study conducted with students found that students who used the distraction method for pain management had significantly higher psychological beliefs scores. ${ }^{10}$ Similarly, this study found a positive weak relationship between the patients' psychological beliefs scores and selfmanagement scores. The correlation between psychological beliefs scores and self-management scores may be due to the fact that individuals who believe the origin of pain is related to psychological factors have stronger personal management. Higher organic beliefs scores cause difficulty in coping with pain and feelings of helplessness. ${ }^{8,10}$ 
Table 3. Comparison of the Patients' Pain-Related Features and PBQ and PCQ Scores

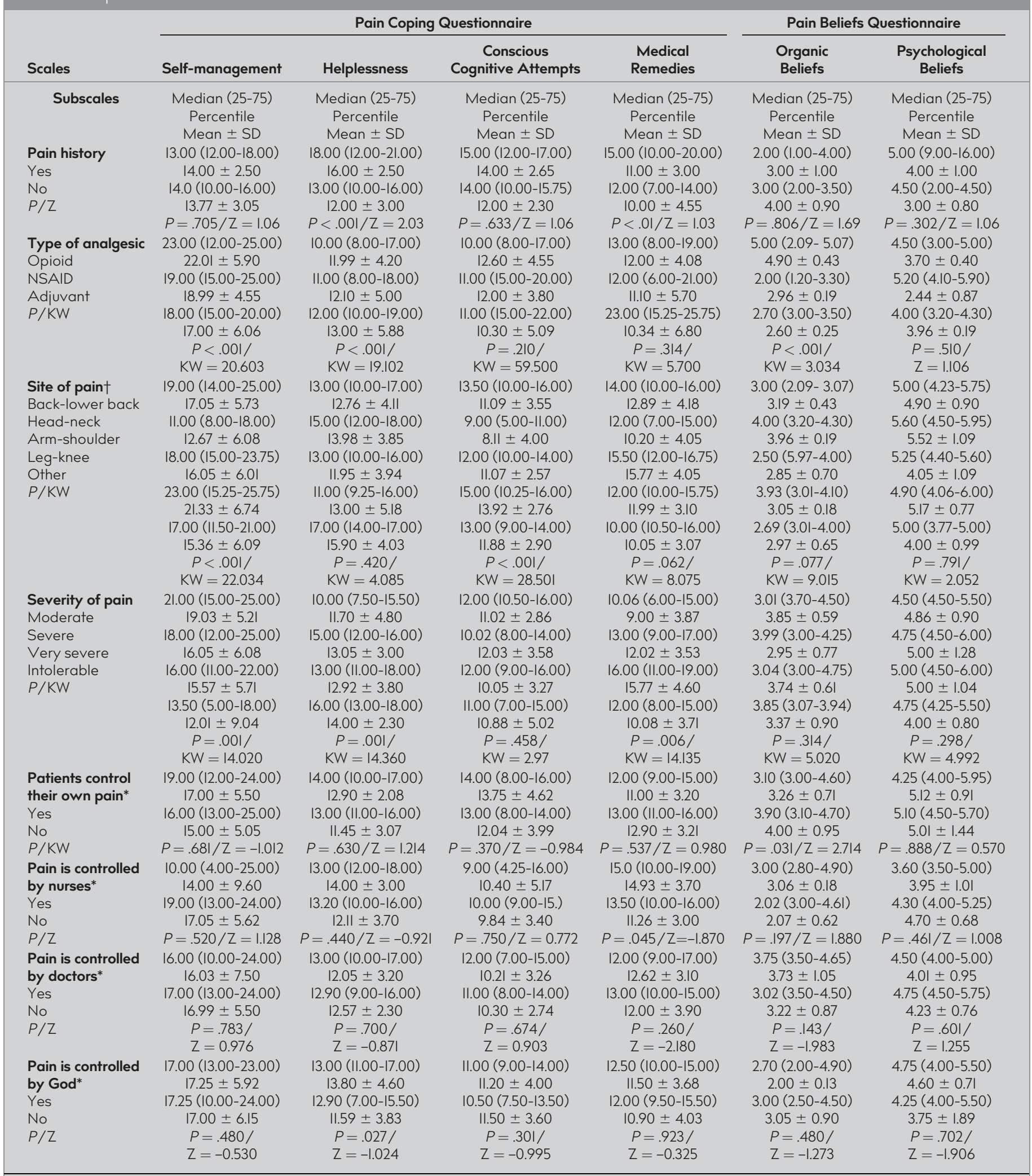

*Mann-Whitney U test.

${ }^{\dagger}$ Kruskal-Wallis test.

The management of pain symptoms, which are common in palliative care patients, significantly affects the quality of care. ${ }^{20}$ Knowing the relationship between pain beliefs and coping with pain may provide substantial benefits in the treatment of pain. Nurses should be aware of individual differences concerning pain and should not consider every patient to be the same. Nurses have critical roles in determining a specific method for each patient in pain management. 8,21 
Beliefs about the nature and treatment of pain may change with multidisciplinary pain management programs based on cognitive-behavioral interventions, and health professionals do not generally take the psychological and cultural components of pain into consideration, but tend to focus on its physiological causes. $^{21,22}$ It is important for nurses as members of healthcare teams to detect the problems of patients related to their pain beliefs in pain management early and to plan nursing interventions that consider patients' pain beliefs. For instance, patients who have low psychological beliefs scores may not benefit from nonpharmacological cognitive-behavioral methods of pain control. ${ }^{8,21}$

The patients' age, marital status, education levels, and income levels had no significant relationships with their organic and psychological pain beliefs scores. The female patients and patients who defined their family members as their caregivers had higher mean psychological pain beliefs scores, but this difference was not statistically significant. Koçoğlu and Özdemir ${ }^{23}$ found that age, education level, and marital status had no relationship with organic beliefs scores. Babadağ et al. ${ }^{18}$ found that females had higher psychological beliefs scores. Higher psychological beliefs scores suggest that female patients use cognitive-behavioral methods more. The female patients had higher self-management, conscious cognitive attempts, helplessness, and medical remedies scores, and this difference was statistically significant. Another study found that female patients had higher helplessness and medical remedies scores than male patients. ${ }^{8}$ The literature emphasizes that cultural expectations, social responsibilities, and social roles affect female patients' coping with pain. ${ }^{24}$ Care support by family members has a positive effect on patients' psychology and eases coping with pain. ${ }^{25}$ This is thought to be the cause of the higher psychological beliefs scores of the patients who defined their family members as their caregivers.

In our study, a significant difference was found between patients' pain history and their scores for helplessness and medical remedies. There are similar results in the literature. ${ }^{8}$ Nurses who have a significant role in pain management can have a positive effect on coping with pain when they consider patients' pain histories while planning nursing interventions.

The patients who used opioids had higher organic beliefs scores than those who did not. A study conducted with patients in algology clinics reported similar results. ${ }^{8}$ The patients who used opioids had higher self-management scores and lower helplessness scores. This is thought to be related to negative and inaccurate beliefs about opioids (fears of their side effects and drug addiction). By giving information about opioids, nurses can have a positive effect on patients' pain beliefs.

The cancer patients had lower self-management scores than the cardiovascular disease patients. This may be due to the fact that cancer causes emotional distress and negative thoughts. The cancer patients had lower conscious cognitive attempts scores than those diagnosed with neurological diseases and had higher medical remedies scores than all the other groups. These results may be due to the fact that patients with cancer consider nonpharmacological methods ineffective for pain management. Healthcare teams should determine the factors that reduce pain tolerance, increase suffering, worsen cancer pain, cause opioid addiction, and negatively affect the pain treatment of cancer patients. ${ }^{25}$

The patients who believed that they control their own pain had lower organic beliefs scores. A significant difference was only found in the helplessness scores of patients who believed that pain is controlled by God. Helmes and Goburdhun ${ }^{26}$ studied patients' beliefs about the control of pain (internal factors, external factors, and luck). They found that more belief in internal factors reduces helplessness, and more belief in external factors and luck increases helplessness. A significant difference was found in the medical remedies scores of the patients who believed that pain is controlled by nurses. ${ }^{26}$ This may be due to the fact that the patients considered nurses to be effective in pain treatment. No significant difference was found in the scale scores of patients who believed that pain is controlled by doctors. Several studies have found that patients who believe that pain is controlled by God feel more helplessness in coping with pain. 8,18 This study's results support the claim that individuals feel helpless against pain when they believe that pain is controlled by external factors. ${ }^{18}$

The cancer patients had lower self-management scores than the cardiovascular disease patients. The cancer patients had lower conscious cognitive attempts scores than those diagnosed with neurological diseases and higher medical remedies scores than all the other groups. This may be due to the fact that the cancer patients had very severe levels of pain, are at risk of depression, and thus have difficulty coping with pain. A significant difference was found between the patients' severity of pain and the subscales of self-management, helplessness, and medical remedies. It is emphasized in the literature that changes in the severity of pain affect coping with pain. ${ }^{27}$ This indicates that diagnosis and severity of pain should be considered in the extensive evaluation of pain.

A significant difference was found between self-management and conscious cognitive attempts scores of the patients with head-neck pain, back-lower back pain, arm-shoulder pain, and leg-knee pain. The results of this study are similar to those in the literature. 8,18 This may be related to the fact that most of the patients in palliative care were cancer patients. Of patients, $50 \%-80 \%$ have been reported to have frequent head-neck pain in their final years and to be unable to cope with this pain by themselves. ${ }^{28}$ The differences between these study results may be due to a variety of sociodemographic and cultural factors. The results of this study are important because they are based on considering sociodemographic and pain-related variables, particularly diagnosis and gender, in the selection of methods of pain management.

In conclusion, higher organic beliefs scores have a positive effect on self-management and conscious cognitive attempts and a negative effect on helplessness. Higher psychological beliefs scores positively affect self-management. The evaluation of the pain, which is one of the main symptoms in palliative care, is the cornerstone of pain management. Pain management involves a variety of difficulties, including lack of effective communication between patients and nurse; psychological, cultural, and social barriers to the diagnosis of pain; and the fact that pain is a multidirectional and subjective concept. Nurses should determine the effects of patients' thoughts, beliefs, and sociodemographic differences on their pain and train patients 
to cope with their pain by establishing effective communication with them. The planning and implementation of nursing pain management interventions should consider the relationships between the pain beliefs and pain coping strategies of patients.

\section{Limitations of the Research}

Since there is only one palliative care clinic in Malatya province, the fact that the study was conducted with 140 patients was considered as a limitation for this study.

Ethics Committee Approval: Ethical committee approval was received from the Inönü University Noninvasive Clinical Research Ethics Committee (approval number: 2019/36-04).

Informed Consent: Both written and verbal informed consents were obtained from all participants who participated in this study.

Peer-review: Externally peer-reviewed.

Author Contributions: Concept - T.M., R.D.; Design - T.M.; Supervision T.M., R.D.; Resources - T.M.; Materials - T.M., R.D.; Data Collection and/or Processing - T.M., R.D., D.A.; Analysis and/or Interpretation - T.M., R.D.; Literature Search - T.M., R.D., D.A.; Writing Manuscript - T.M., R.D.; Critical Review - T.M., R.D., D.A.

Conflict of Interest: The authors have no conflicts of interest to declare.

Financial Disclosure: The authors declared that this study has received no financial support.

\section{REFERENCES}

I. Bhattacharya P, Dessain SK, Evans TL. Palliative care in lung cancer: When to start. Curr Oncol Rep. 2018;20(II):90.

2. Yürüyen $M$, Tevetoğlu IÖ, Tekmen $Y$, et al. Prognostic factors and clinical features in palliative care patients. Konuralp Tıp Dergisi. 2018;10(I):74-80

3. Bhatnagar S, Gupta M. Integrated pain and palliative medicine model. Ann Palliat Med. 2016;5(3):196-208.

4. Allsop MJ, Taylor S, Mulvey MR, et al. Information and communication technology for managing pain in palliative care: A review of the literature. BMJ Support Palliat Care. 2015;5(5):48I-489.

5. McGuire DB, Kaiser KS, Haisfield-Wolfe ME, et al. Pain assessment in non-communicative adult palliative care patients. Nurs Clin N Am. 2016;5I(3):397-431.

6. Hocaoglu A, Berk HÖS, Ketenci A, et al. The Study of Reliability and Validity of the Pain Coping Inventory. Agri. 2019;3I(I):32-4l. [CrossRef]

7. Cáceres-Matos R, Gil-García E, Cabrera-León A, et al. Factors that influence coping with chronic noncancer pain in European countries: A systematic review of measuring instruments. Pain Manag Nurs. 2020;2I(2):123-133.

8. Babadağ B, Alparslan GB, Güleç S. The relationship between pain beliefs and coping with pain of algology patients. Pain Manag Nurs. 2015;16(6):910-919.
9. Peres MF, Lucchetti G. Coping strategies in chronicpain. Curr Pain Headache Rep. 20I0;14(5):331-338.

10. Babadağ B, Alparslan GB. The pain beliefs of nursing students. Sted. 2017;26(6):244-250.

II. Birge Mollaoğlu ÖA. Pain beliefs of patients and the nonpharmacological methods they use to manage the pain. Ağrı. 2018;30(2):84-92.

12. Azevedo LF, Sampaio R, Dias CC, et al. Portuguese version of the pain beliefs and perception inventory: A multicenter validation study. Pain Pract. 2017;17:808-819.

13. Anderson F, Downing GM, Hill J, et al. Palliative performance scale (PPS): A newtool. J Palliat Care. 1996;12(I):5-II.

14. Edwards LC, Pearce SA, Turner-Stokes $L$, et al. The pain beliefs questionnaire: An investigation of beliefs in the causes and consequences of pain. Pain. 1992;5I(3):267-272.

15. Berk HO. The experience of chronic pain and pain beliefs: The Turkish validation study of the pain beliefs questionnaire [in Turkish]. Unpublished doctorate thesis, Istanbul Universitesi Sosyal Bilimler Enstitüsü, Istanbul, Turkey, 2006.

16. Kleinke $\mathrm{CL}$. How chronic pain patients cope with pain: Relation to treatment outcome in a multidisciplinary pain clinic. Cogn Ther Res. 1992;6:669-685.

17. Tıglı A, Günebakan Ö, Toslalı Z, et al. The relationship between the level of cognition, pain and body awareness in geriatric persons. Sağlık ve Toplum. 2019;29(2):86-91.

18. Babadağ B, Balcı Alparslan G, Güleç S. Coping with the pain of elderly pain patients: Nursing approach. Agrı. 2017;29(2):55-63.

19. Madenci E, Herken H, Yağız E, et al. Depression levels and the ways of coping with pain in patients with chronic pain and fibromyalgia syndromes. Türk Fiz Tip Rehab Derg. 2006;52(1):19-21.

20. Chang VT, Sorger B, Rosenfeld KE, et al. Pain and palliative medicine. J Rehabil Res Dev. 2007;44(2):279.

21. WalshDA RJ. Pain beliefs and perceived physical disability of patients with chronic low back pain. Pain. 2002;97(I):233I.

22. Krupić $F$, Custović $S$, Jašarević $M$, et al. Ethnic differences in the perception of pain: A systematic review of qualitative and quantitative research. Med Glas. 2019;16(1):108-||4.

23. Koçoğlu D, Özdemir L. The relation between pain and pain beliefs with socio-demographic-economic characteristics at the adult population. J Turk Soc Algol. 20ll;23(2):64-70.

24. Pieretti S, DiGiannuario A, DiGiovannandrea R, et al. Gender differences in pain and its relief. Ann Ist Super Sanita. 2016;52(2):184-189.

25. Dedeli Ö, Karadeniz G. An integrated psychosocial-spiritual model for cancer pain management. Agri. 2009;2I(2):45-53.

26. Helmes E, Goburdhun A. Cognitions related to chronic pain: Revision and extension of the cognitive evaluation questionnaire. Clin $\mathrm{J}$ Pain. 2007;23(1):53-61.

27. Geisser E, Robinson ME, Riley JL. Pain beliefs, coping, and adjustment to chronic pain: Let's focus more on the negative. Pain Forum. 1999;8(2):161-168.

28. Babgi AA. Pain coping behaviors of Saudi patients suffering from advanced cancer: A revisited experience. Asian Pacific J Cancer Preven. 2010;11:103-106. 